Citation: M. Repetto (2020) La scuola come polo educativo e socio-culturale nell'era pandemica del COVID-19. Media Education 11(2): 49-59. doi: 10.36253/me-9673

Received: September, 2020

Accepted: October, 2020

Published: December, 2020

Copyright: ( $) 2020$ M. Repetto. This is an open access, peer-reviewed article published by Firenze University Press (http://www.fupress.com/me) and distributed under the terms of the Creative Commons Attribution License, which permits unrestricted use, distribution, and reproduction in any medium, provided the original author and source are credited.

Data Availability Statement: All relevant data are within the paper and its Supporting Information files.

Competing Interests: The Author(s) declare(s) no conflict of interest.

\section{La scuola come polo educativo e socio-culturale nell'era pandemica del COVID-19}

\author{
School as educational and socio-cultural hub in the pandemic \\ age of COVID-19
}

\author{
Manuela Repetto \\ Dipartimento di eccellenza di Filosofia e Scienze dell'Educazione, Università degli Studi \\ di Torino \\ E-mail: manuela.repetto@unito.it
}

\begin{abstract}
The school and the institutions of the local community play a complementary role on how the educational system currently works, as well as on its desired transformation. In light of the changes that the pandemic imposes on a structural and regulatory level, it is necessary to reorient reflection and research also on pedagogical models that can facilitate a transformation process already underway before the pandemic and that the current situation brings to the fore. One way could be to favor the contamination between formal knowledge and the repertoire of knowledge and educational practices spread throughout the territory. In this mutual contamination, the role of digital technologies and educational media appears crucial, which will be explored in this contribution by defining, through a taxonomic model, some scenarios on the possible educational initiatives that arise from the alliance between school and territory. These scenarios, in addition to offering solutions to address the risks posed by the pandemic situation, represent an opportunity to innovate the educational system.
\end{abstract}

Keywords: formal and informal learning, media education, technology enhanced learning, educational community.

Riassunto. La scuola e le istituzioni del territorio rivestono un ruolo complementare nell'attuale funzionamento del sistema educativo, così come nella sua auspicata trasformazione. Alla luce dei cambiamenti che la pandemia impone a livello strutturale e normativo, occorre riorientare la riflessione e la ricerca anche sui modelli pedagogici che possono agevolare un processo di trasformazione già in atto prima della pandemia e che la situazione attuale riporta in primo piano. Una via da percorrere può essere quella di favorire la contaminazione fra il sapere formale e il repertorio di conoscenze e pratiche educative diffuso sul territorio. In questa contaminazione reciproca, appare cruciale il ruolo delle tecnologie digitali e dei media educativi, che verrà esplorato in questo contribuito definendo, attraverso un modello tassonomico, alcuni scenari sulle possibili iniziative educative che scaturiscono dall'alleanza fra scuola e territorio. Tali scenari, oltre ad offrire delle soluzioni per affrontare i rischi posti dalla situazione pandemica, rappresentano un'opportunità per innovare il sistema educativo.

Parole chiave: apprendimento formale e informale, media education, apprendimento supportato dalle tecnologie, comunità educante. 


\section{INTRODUZIONE}

Quello educativo può essere considerato un sistema duale formato, da una parte, dal modello istituzionale di istruzione formale, ossia da tutto ciò che attiene alla scuola e che viene da essa generato all'interno degli istituti scolastici; dall'altra parte, dall'offerta educativa del territorio, rappresentata dall'insieme di iniziative promosse da associazioni, imprese, dalla comunità nel suo insieme e da tutte quelle organizzazioni dedite a promuovere forme di apprendimento informale e non formale, che con la scuola possono interagire e collaborare. La scuola e il territorio si possono riconnettere con azioni educative congiunte (Repetto, 2013), in modo che la scuola possa integrare e contaminare il sapere formale con le conoscenze costruite all'esterno, mentre il territorio può assorbire e formalizzare i propri processi di conoscenza informali ed estemporanei Questa contaminazione reciproca, da sempre auspicata da chi fa ricer$\mathrm{ca}$ in campo educativo, si rende sempre più impellente, anche alla luce delle condizioni attuali e dei cambiamenti che la pandemia del COVID-19 impone (Cahapay, 2020), soprattutto in relazione ad esigenze come quella del distanziamento, della creazione di piccoli gruppi stabili e circoscritti, del decentramento delle attività didattiche in strutture esterne alla scuola laddove gli spazi fossero limitati, del mantenimento della dimensione sociale e collaborativa dell'apprendimento anche in situazioni di isolamento forzato dovuto alle quarantene o alla malattia.

Attualmente il disallineamento fra scuola è territorio, che in Italia così come altrove è ancora molto forte, è tale da far apparire il sistema scolastico costantemente inadeguato, rigido e arretrato. La cultura dominante si basa sul presupposto che gli studenti vadano istruiti e che l'istruzione possa essere impartita soltanto attraverso il sistema scolastico formale, che inevitabilmente consegna alla società studenti sempre più impreparati, con competenze poco spendibili e incapaci di inserirsi appieno nel mondo del lavoro. Sebbene un'educazione formale rigorosa sia fondamentale, essa, per poter attivare processi di apprendimento più efficaci e che valorizzino le potenzialità di ogni studente, dovrebbe essere integrata con esperienze formative concrete ed autentiche condotte al di fuori della scuola, inglobando e integrando nei curricoli d'istituto ciò che può contribuire a potenziare l'offerta formativa scolastica tradizionale. Queste esperienze extrascolastiche o integrate, dal canto loro, dovrebbero mantenere una forte connessione con le attività scolastiche, perché possano sempre essere ricondotte al sapere e alla riflessione e, allo stesso tempo, contribuire a generare nuove e virtuose pratiche scolastiche.
Con il presente contributo si intende, dopo aver analizzato gli elementi di discontinuità tra istruzione formale e apprendimento non formale e informale, riflettere su come la scuola, assumendo il ruolo di connettore socioculturale oltre che educativo, può integrare la propria offerta formativa ed espanderla con le numerose iniziative generate dalla comunità locale. A partire dall'analisi di alcuni modelli pedagogici che si fondano sulle interconnessioni tra la scuola e il territorio, si propone un modello tassonomico ispirato all'approccio sistemico ecologico per definire e distinguere le possibili iniziative educative che scaturiscono dalle alleanze fra scuola e territorio e che lasciano intravvedere dei possibili orizzonti su come gestire i processi educativi nell'era pandemica del COVID-19.

\section{LA RELAZIONE FRA ISTRUZIONE FORMALE E APPRENDIMENTO INFORMALE}

Le ultime decadi sono state caratterizzate, in Italia ed ovunque, da una progressiva quanto inarrestabile trasformazione del sistema educativo, che gli ultimi accadimenti relativi alla pandemia hanno fortemente accelerato e reso ancora più impellente. Questa trasformazione si traduce nella tendenza a rendere i processi di apprendimento ed insegnamento più flessibili (Daniel, 2020; De Marco, 2020; Galdieri et al., 2020; Selwyn, 2011) in termini di spazi (Marcarini, 2014), di approcci metodologici e di potenzialità educative. Le aule tradizionali, alla luce delle condizioni socio-economiche e dei recenti progressi tecnologici della società della conoscenza, appaiono sempre più inadeguate (Tosi, 2019), mentre le potenzialità pedagogiche insite in un ambiente scolastico non limitato ai confini dell'aula tradizionale, cominciano ad essere sempre più esplorate ed incoraggiate. Imms et al. (2017) definiscono l'ambiente di apprendimento innovativo con un concetto che racchiude diversi aspetti complementari: esso comprende non soltanto l'innovazione sul versante dello spazio fisico per promuovere forme di flessibilità dei processi di insegnamento ed apprendimento, ma anche le innovazioni pedagogiche, che pongono lo studente al centro e che promuovono la personalizzazione dell'apprendimento, incentivano i processi collaborativi e valorizzano l'esperienza di apprendimento anche in un'ottica pluri- e interdisciplinare. In letteratura emergono alcune prospettive epistemologiche (Mulcahy et al., 2015) per comprendere la relazione tra l'ambiente fisico e le innovazioni pedagogiche. Una di queste prospettive, quella relazionale, definisce spazi e pratiche socio-pedagogiche come strettamente interconnessi, in un rapporto dialettico secondo cui spazi e 
pratiche si informano e si influenzano reciprocamente, in modo tale che l'ambiente non è dato ma, piuttosto, si viene a costruire nel divenire delle azioni sociali e didattiche. Secondo questa prospettiva quindi, non sono gli ambienti predefiniti e già dotati di infrastrutture a far emergere le pratiche innovative, in maniera deterministica; piuttosto questi ambienti si vengono a definire nel quadro di relazioni più complesse che, adottando un approccio socio-materialista, comprende elementi tangibili quali gli arredi, gli schermi, le pareti, i dispositivi tecnologici e le persone, così come elementi intangibili quali le credenze pedagogiche, le emozioni, i sistemi valoriali (Acton, 2017). È nell'ambito di questo complesso intreccio di relazioni che va, dunque, ridefinito e costruito l'ambiente di apprendimento, modificandolo e riprogettandolo alla luce degli esiti che si ottengono dalle pratiche didattiche sperimentate. Ed è nell'ambito di questo quadro, che emerge la necessità di conciliare gli elementi di discontinuità fra l'istruzione formale e quella informale.

L'eccessiva enfasi sull'istruzione formale, oltre a minare la vita scolastica degli studenti, va a detrimento dell'intera comunità e del capitale umano (Sahlberg, 2011). Gli studenti cui viene proposto un modello didattico tradizionale, tendono a percepire le esperienze formative extrascolastiche come più motivanti e coinvolgenti, seppur queste non vengano in alcun modo riconosciute né, tanto meno, valorizzate dagli insegnanti. Le esperienze informali tendono infatti a differire da quelle scolastiche in termini di finalità, di rilevanza e di utilità, seppur dovrebbero essere equamente legittimate in quanto anch'esse influiscono sulle identità, sugli interessi, sui vissuti e sullo sviluppo delle competenze degli studenti (Bevan et al., 2013; Repetto, 2013).

Già tre decenni or sono, Resnick (1987) individuava quattro fattori che differenziavano l'apprendimento formale da quello extrascolastico, sia informale che non formale. La prima delle contrapposizioni riguarda la forma dominante di apprendimento, che a scuola è individuale, mentre fuori tende ad essere socialmente condivisa. Se infatti in classe la maggior parte delle attività, come i compiti, le spiegazioni e le verifiche, sono incentrate sull'individuo, e anche le attività fatte in gruppo sfociano, in ultima analisi, in giudizi e valutazioni espressi sui singoli, nella vita quotidiana e in quella lavorativa e ricreativa, l'abilità del singolo tende ad intrecciarsi con quella di altre persone facenti parte dello stesso ambito. La classe scolastica tradizionale non può infatti essere considerata una comunità, tanto che gli studenti appaiono come tante monadi i cui singoli contributi sono irrilevanti per la classe nel suo insieme; al contrario, nei gruppi esterni gli individui sono legati agli altri in relazioni reciproche, cosicché ciò che viene conseguito individualmente ha ripercussioni su tutto il gruppo (Roth \& Lee, 2006): la conoscenza costruita socialmente non è incapsulata e fine a se stessa, ma è significativa e utile per tutti (Engeström, 1991). Un secondo elemento di contrasto è il sapere di tipo teorico e speculativo su cui si fonda principalmente l'istruzione formale, contrapposto al sapere più pratico dei percorsi informali, basato sulla manipolazione di strumenti. Un terzo elemento di discontinuità riguarda il pensiero simbolico, più astratto e avulso dalle situazioni reali, diffuso a scuola, cui si contrappone il ragionamento contestualizzato condotto al di fuori di essa. Il quarto ed ultimo elemento concerne l'apprendimento generalizzato che contraddistingue la scuola, contrapposto a quello invece centrato su competenze specifiche che pervade all'esterno. I quattro suddetti elementi di discontinuità tra la dimensione scolastica e quella extrascolastica individuati da Resnick si fanno oggi ancora più marcati, con effetti negativi sul rendimento degli studenti così come esso viene percepito dai docenti, che per stimarlo tendono a basarsi su elementi più comportamentali che cognitivi (Singal \& Swann, 2011). Ma gli effetti sono ancora più devastanti se si considera come gli studenti vivono le proprie esperienze scolastiche avvertendole come totalmente sconnesse dalla vita reale.

Ad alimentare questa discontinuità contribuisce anche la diffidenza che l'istituzione scolastica talvolta mostra nei confronti delle figure educative (formatori, genitori, volontari) che promuovono iniziative formative extrascolastiche, considerate come amatoriali e improvvisate, prive di metodo e incapaci di contribuire sistematicamente alla formazione degli studenti (Chrzanowski et al., 2010). Peraltro, anche laddove le istituzioni scolastiche manifestino l'intenzione di promuovere iniziative all'esterno, esse tendono ad imporre una concezione di apprendimento che solitamente mutuano dallo stesso contesto scolastico (Resnick, 1987), senza lasciarsi contaminare da idee ed approcci alternativi, in quanto non istituzionalizzati. Tali approcci, al contrario, potrebbero fungere da stimolo (Hull \& Schultz, 2001) per un profondo rinnovamento della scuola.

Le molteplici traiettorie del percorso formativo che ogni studente attraversa in maniera lineare e pressoché rigida dentro la scuola e in modo caotico al di fuori di essa, senza spesso riuscire a trovare punti di contiguità fra di esse, potrebbero forse ricongiungersi se la scuola riuscisse a connettere le esperienze informali che gli studenti vivono nella vita quotidiana, sul loro territorio, e le attività formali proposte in classe. La situazione pandemica attuale, seppur introduca ulteriori elementi di complessità, rappresenta un'opportunità unica per provare a 
tracciare nuove traiettorie avvalendosi delle esperienze che si possono attivare sul territorio.

\section{TECNOLOGIE E MEDIA EDUCATIVI COME CONNETTORI}

La società attuale necessita di persone autonome, creative, intraprendenti, capaci di risolvere problemi, in grado di muoversi in situazioni collaborative e in possesso di altre analoghe competenze che non possono essere acquisite soltanto in classe, ma che dovrebbero attingere anche all'esterno, attraverso nuove esperienze formative provenienti dall'intera comunità (Abbott, 2014). In questo quadro, la scuola potrebbe fungere, in primo luogo, da aggregatore, da "hub" delle molteplici occasioni formative che si trovano sul territorio (Paone, 2020). Dopo averle sondate e selezionate, la scuola potrebbe dunque diventare un punto di riferimento per il proprio territorio, un polo educativo, sociale e culturale che conferisce senso a queste occasioni, da una parte assimilandole nelle proprie pratiche educative, dall'altra contribuendo a farle evolverle e a renderle più organiche e durature, in una prospettiva di formazione integrata. Le politiche educative, con il decreto sull'autonomia scolastica, spingono gli istituti scolastici in questa direzione, invitandoli a perseguire una duplice linea formativa, quella verticale e quella orizzontale, come si evince anche dalle Indicazioni nazionali (AAVV, 2012). La linea verticale fa riferimento all'esigenza di prevedere per gli studenti una formazione che si sviluppi lungo tutto l'arco della vita; quella orizzontale si incentra sulla necessità, per la scuola, di attivare collaborazioni con tutti gli attori extrascolastici con valenza educativa, a cominciare dalla famiglia. Aprendosi alle famiglie e alla comunità di appartenenza nel suo complesso, la scuola esercita appieno la propria autonomia, diventando a tutti gli effetti una comunità educativa integrata, costruttrice di "alleanze" con tutti i possibili soggetti del pubblico e del privato, con i quali instaura legami stabili, avvia progettualità comuni, condivide, oltre alle pratiche e alle procedure organizzative, prospettive di senso e di cambiamento (Save the Children Italia, 2012).

Nella costruzione delle suddette alleanze con i vari attori della comunità, le tecnologie e i media educativi assumono il ruolo chiave di connettore: essi infatti supportano, in primo luogo, l'attività di networking fra la scuola e gli altri soggetti presenti sul territorio e altrove, facilitando la comunicazione e sostenendo le dinamiche collaborative e organizzative. Ma tecnologie e media educativi rivestono anche e soprattutto una valenza didattica: la cornice spazio-temporale delle atti- vità didattiche proponibili agli studenti può, grazie alla mediazione delle attuali tecnologie, dilatarsi a dismisura, moltiplicando, intersecando ed estendendo le loro traiettorie formative. Le tecnologie, infatti, ampliano gli spazi laddove i confini delle aule scolastiche, delle sale dei musei, degli uffici delle aziende, dei palchi dei teatri e dei luoghi fisici di tutte le altre agenzie educative, limitano le attività degli studenti e, in un epoca di distanziamento, non le rendono del tutto fattibili.

Allo stesso modo, le tecnologie dilatano i tempi di svolgimento delle attività, che non sono più vincolate al calendario scolastico, agli orari di visita del museo, a quello degli uffici, dello spettacolo teatrale, o a quello di apertura delle biblioteche.

Tuttavia, queste stesse tecnologie, laddove il loro impiego è discontinuo e frammentario, soprattutto se l'uso personale si discosta eccessivamente con l'uso che se ne fa a scuola, rischiano di diventare un elemento di discontinuità e di ulteriore divario, anziché di connessione. Le ricerche condotte sull'uso dei social media da parte dei giovani al di fuori dalla scuola mostrano, infatti, come le pratiche di utilizzo siano differenti da quelle scolastiche in termini di modalità, intenti e contenuti (Gee, 2003; Ito et al., 2009; Roth \& Erstad, 2013). Una delle sfide attuali che docenti ed educatori dovrebbero porsi, anche alla luce degli ulteriori divari che si sono generati durante il lockdown dovuti alla mancanza di tecnologie o di connessione adeguata che hanno impedito a molti studenti di partecipare alle attività di didattica a distanza (Livari et al., 2020), consiste proprio nel cercare di comprendere e di attuare delle interconnessioni che le tecnologie possono attivare fra le pratiche scolastiche formali e quelle informali. In questo modo, si può puntare a ricomporre il mosaico di conoscenze che gli studenti costruiscono nelle loro traiettorie fra casa, scuola e territorio. Inoltre, coinvolgendo la comunità e delegando ad esperti esterni alla scuola la realizzazione di nuovi percorsi educativi fruibili attraverso tecnologie e media educativi, si può supportare la scuola nell'arduo compito di continuare a garantire un'istruzione di qualità anche in una situazione di emergenza come quella che stiamo vivendo (Iyengar, 2020).

Se dunque le tecnologie e i media possono essere considerate alla stregua di strumenti culturali cruciali per integrare l'apprendimento formale con quello informale, ci si dovrebbe interrogare su come impiegarle didatticamente e a quali teorie occorre fare riferimento. Uno degli approcci che dal punto di vista pedagogico meglio incarna il valore che viene conferito all'apprendimento informale e non formale e che promuove l'idea di connetterlo con il sistema di istruzione formale, facendo ricorso anche alle tecnologie digitali, è l'apprendimento 
connettivo (Connected Learning) (Antero, 2014; Ito et al., 2013), che si basa sulla concezione di un apprendimento socialmente situato, mosso dall'interesse personale e orientato verso opportunità educative, economiche o politiche. Lapprendimento connettivo si attua quando lo studente viene posto nelle condizioni di coltivare un interesse o una passione con l'aiuto dei pari o di figure educative adulte e di coniugare questo interesse con il profitto scolastico, con il successo lavorativo o con l'impegno civico. I sostenitori di questo approccio riconoscono le potenzialità delle tecnologie digitali nel connettere casa, scuola, comunità e gruppi di pari e nel promuovere il dialogo intra- e inter-generazionale, fondato su interessi comuni. In particolare, le tecnologie assolvono alcune funzioni attraverso le quali potenziano le opportunità di apprendimento connettivo, promuovendo la partecipazione attiva e molteplici forme di espressione personale, valorizzando le diversità e minimizzando le inequità dei gruppi a rischio di povertà educativa (Garcia, 2014; Nussbaum-Beach \& Ritter, 2011; Siemens, 2004). Nell'esaminare il ruolo che le tecnologie rivestono nella vita quotidiana degli studenti e dei giovani, i fautori dell'apprendimento connettivo si avvalgono del concetto di ecologia come metafora per riferirsi ai contesti e alle loro interconnessioni che fanno da cornice allo studente che cresce e si forma.

\section{UN MODELLO ECOLOGICO PER RAPPRESENTARE LE CONNESSIONI FRA SCUOLA E TERRITORIO}

Recuperando in chiave pedagogica il noto modello ecologico di Bronfenbrenner $(1979 ; 1987 ; 1990)$ e tenendo conto dei principi dell'apprendimento connettivo illustrati nel paragrafo precedente, si può collocare al centro del modello lo studente in crescita, il cui apprendimento è influenzato dalle quattro strutture concentriche (micro, meso, eso e macro) e dalle loro interconnessioni (Figura 1). Se le entità che appartengono a questi sistemi - la scuola, la famiglia e la comunità - interagiscono e collaborano sinergicamente rispondendo ai suoi bisogni formativi e sociali, allora lo studente non può che trarre il massimo giovamento dalle relazioni supportive che tali entità riescono ad instaurare con lui.

Se si assume un'ottica ecologica e sistemica, l'apprendimento può dunque essere considerato come un processo che scaturisce dall'interazione di fattori individuali, sociali e culturali rintracciabili nelle quattro strutture definite da Bronfenbrenner. Nell'attribuire alla scuola il ruolo di polo educativo come descritto nel par. 3, un modello siffatto può fungere da riferimento per inquadrare le dinamiche che la scuola può attiva-

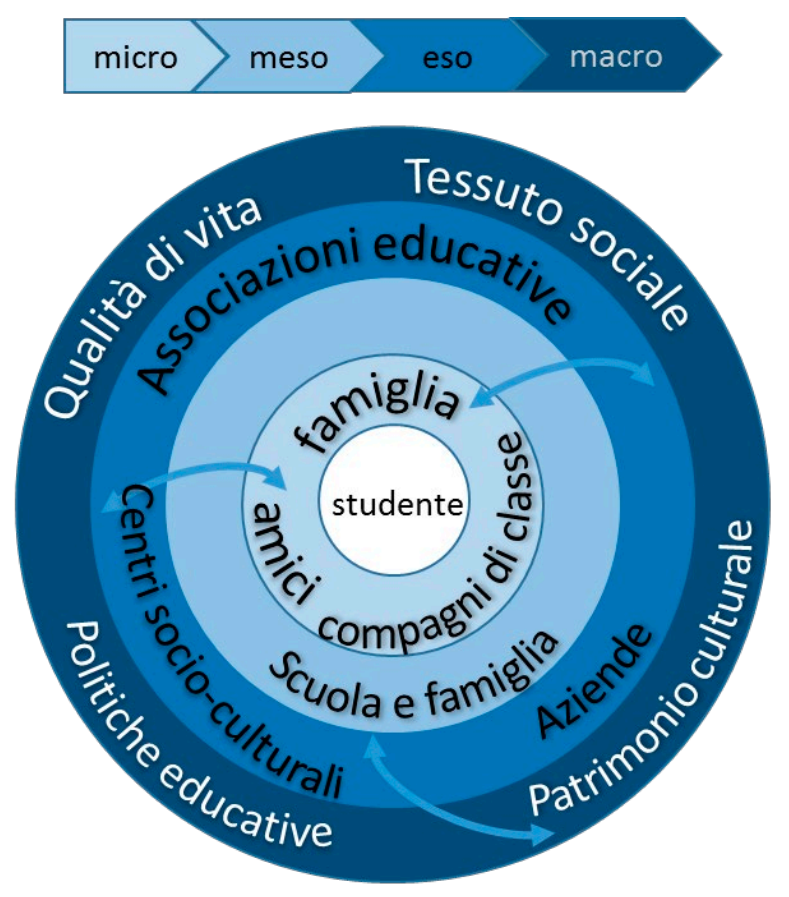

Figura 1. Una rilettura in chiave pedagogica degli elementi che compongono il modello ecologico di Bronfenbrenner.

re in modo sistematico con le altre agenzie educative e con le varie entità della comunità circostante. Considerando le possibili forme di collaborazione e le tipologie di progetti didattici che la scuola può attivare con queste entità esterne, può risultare funzionale sovrapporre a questi cerchi concentrici due assi ortogonali che rappresentano, rispettivamente, il ruolo specifico assunto dalla scuola, che indica in che modo essa si relazioni con i vari soggetti del territorio per promuovere attività didattiche congiunte (asse orizzontale), e l'ambiente di apprendimento prevalente attraverso cui queste attività hanno luogo, le cui caratteristiche sono state descritte nel paragrafo 2 (asse verticale). L'asse orizzontale (Figura 2) riporta ad un estremo, quello destro, la "scuola polivalente" e, all'altro estremo, "la scuola reticolare"; l'asse verticale contrappone, invece, all' "ambiente online" quello "fisico". Ai due assi ortogonali si aggiunge un terzo asse, il "livello di incidenza", che esprime l'impatto delle iniziative educative in relazione ai livelli micromeso-macro.

Considerando l'estremo destro dell'asse orizzontale, con il concetto di scuola polivalente si fa riferimento all'istituto scolastico che amplia ed accentra la propria offerta formativa, sia in senso temporale che spaziale, moltiplicando le proprie funzioni in ragione dei bisogni dei propri studenti e della popolazione presente nel territorio. La scuola dunque si appropria di quelle fun- 


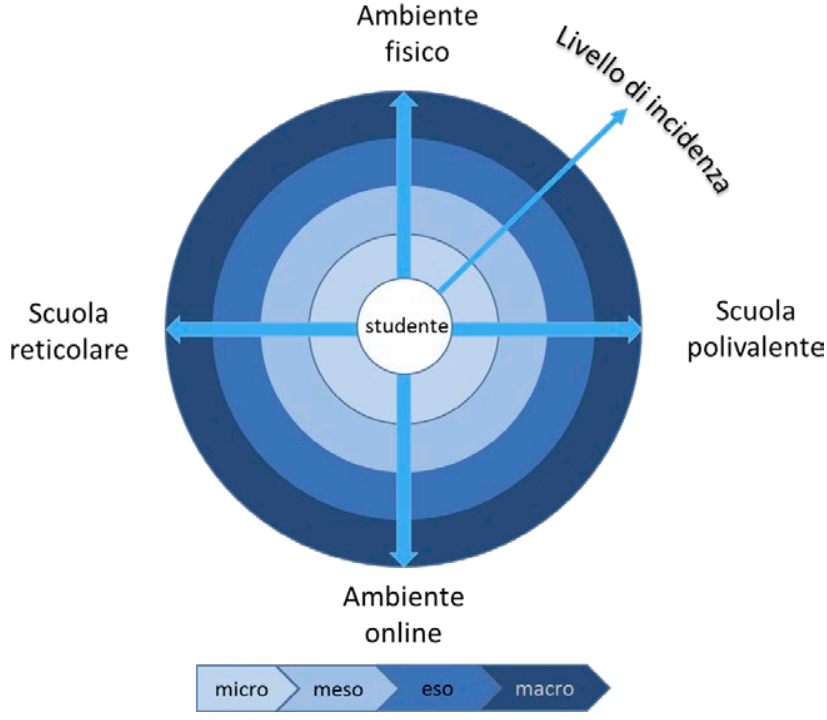

Figura 2. Un approccio sistemico per rappresentare la scuola come polo educativo e socio-culturale.

zioni educative che solitamente vengono assolte da strutture al di fuori e, allo stesso tempo, si apre al territorio accogliendo nelle proprie strutture la cittadinanza come potenziale utenza a cui rivolgere la propria offerta: in questo modo la scuola propone percorsi extrascolastici, gestisce al proprio interno servizi che sono appannaggio di altri enti locali (biblioteche, centri ricreativi, atelier, teatri, musei) e promuove iniziative culturali quali seminari, cineforum, mostre, concerti e quant'altro. Alla realizzazione di queste iniziative temporanee o permanenti collaborano, oltre al personale scolastico, professionisti, cittadini, associazioni, ma anche gli stessi studenti.

All'estremo opposto dell'asse si contrappone la scuola reticolare, ossia la scuola che si delocalizza e che collabora e costruisce reti e partenariati con i soggetti del territorio i quali, facendosi promotori o proponendosi anche come recettori di iniziative didattiche, accolgono gli studenti nei propri spazi o predispongono le proprie strutture per svolgere azioni educative rivolte alla popolazione studentesca, così come a tutta la cittadinanza. In questo modo, la scuola, assieme ai soggetti pubblici e privati che rappresentano la comunità educante, si decentra e si distribuisce capillarmente su tutto il territorio, allargando la propria offerta formativa in un modo alternativo e del tutto opposto rispetto a quello della scuola polivalente. Questo modello organizzativo appare più confacente alla situazione pandemica attuale, in quanto la scuola non richiama la popolazione al proprio interno né concentra tutte le attività e i servizi nelle proprie strutture; al contrario, la scuola reticolare si appoggia al territorio e alle sue strutture, delegando ad esse anche alcune delle funzioni educative che le sono proprie.

Le iniziative congiunte fra scuola e territorio possono tradursi nella creazione di nuove strutture o nell'allocazione di quelle già esistenti per lo svolgimento delle relative attività; tali strutture, siano esse collocate dentro la scuola (la biblioteca civica, l'atelier, l'auditorium) o siano esse, considerando il territorio, situate esternamente (lo spazio per l'apprendistato dell'impresa locale, la saletta del cinema, gli spazi del centro ricreativo), occupano un ben identificato spazio fisico, rappresentato all'estremo superiore dell'asse verticale (ambiente fisico). All'estremo opposto si collocano quelle iniziative che hanno l'ambiente online come scenario principale per lo svolgimento delle relative attività didattiche, sia esso un portale, uno strumento per la comunicazione, un social media o una realtà virtuale. Le attività didattiche possono essere svolte online o in presenza, ma necessitano di un ambiente di supporto allestito appositamente sul web per scaricare le risorse, comunicare con il docente, collaborare con i compagni o con esperti di altre istituzioni, per creare dei prodotti digitali o condurre delle sperimentazioni, individualmente o in gruppo.

I due assi suddividono l'area composta dalle forme circolari concentriche in quattro quadranti, ciascuno dei quali è dunque delimitato da due semiassi e da una serie di archi paralleli. Le aree incorniciate da questi archi possono essere impiegate per posizionare le iniziative congiunte scuola - territorio, esprimendo in questo modo la loro portata e la loro incidenza sul territorio. Queste iniziative educative assegnano allo studente, che contribuisce attivamente alla costruzione dell'ambiente in cui vive e le cui possibili azioni hanno ricadute a livello di micro, meso, eso o macrosistema, il ruolo di protagonista dinamico. I progetti didattici fortemente innovativi che influiscono a livello politico, che hanno delle ripercussioni economiche, sociali o ambientali sul territorio o che incidono su atteggiamenti e disposizioni valoriali, si collocano negli spicchi più esterni, mentre quelli che hanno un impatto più limitato e circoscritto si situano nelle aree più interne.

Nel valutare l'incidenza di tali iniziative in questi termini, non si intende formulare dei giudizi di valore, stimando come più apprezzabili le iniziative che agiscono a livello macro rispetto a quelle efficaci a livello micro; bensì ci si propone unicamente di differenziare gli ambiti e le strutture interessate dagli effetti di queste iniziative, alcune delle quali incidono più profondamente sugli studenti e sulle loro cerchie più ristrette, mentre altre hanno un effetto più diffuso ma meno dirompente e rapido, coinvolgendo però fasce più ampie della cittadinanza. Questa terza varabile, l'incidenza, contribuisce 
a circoscrivere e a definire le possibili iniziative inscrivibili in ciascuno dei quattro quadranti.

\section{ALLEANZE FRA SCUOLA E TERRITORIO NELLA SITUAZIONE ATTUALE}

Un modello siffatto, strutturato in quattro quadranti e in dodici spicchi, può essere utilizzato per classificare qualsiasi iniziativa educativa sulla base del modello adottato dalla scuola (iniziativa integrata nell'ambiente scolastico o promossa sul territorio), dell'ambiente di apprendimento prevalente (online o in presenza) e del livello di incidenza dell'iniziativa (coinvolgimento di una classe, di una piccola comunità o di un target più ampio della popolazione (si veda Figura 3). Tale modello, inoltre, può orientare il progettista, l'educatore o l'insegnante nell'individuare le coordinate entro cui circoscrivere l'iniziativa che intende proporre e nel posizionarsi in quella più appropriata in relazione al livello di emergenza. Ovviamente la suddivisione in quadranti e spicchi ha la funzione di fornire una rappresentazione semplificata di una realtà molto complessa che inquadra la scuola nelle sue relazioni con il territorio: i confini tra un quadrante e l'altro e tra i vari spicchi non sono tracciati in modo netto, ma sono da considerarsi permeabili; le variabili contestuali, dettate anche dalle condizioni legate all'emergenza, possono trasformare le iniziative già in essere facendole transitare in modo fluido da un quadrante all'altro o attraverso i vari spicchi.
Il primo quadrante, delimitato dai semiassi ambiente fisico/ scuola polivalente, comprende le iniziative gestite dalle scuole nei propri edifici, con il supporto di vari soggetti territoriali. Da una rassegna e da una mappatura condotta negli ultimi anni in merito alle esperienze educative più recenti proposte dalla scuola italiana e realizzate all'interno delle proprie strutture, si possono citare a titolo esemplificativo, a livello micro, lo sportello di ascolto o di orientamento, il laboratorio didattico, l'atelier, i programmi extracurricolari (musicali, sportivi, artistici), i progetti tematici interdisciplinari, i progetti di inclusione socio-educativa e di intercultura. A livello meso si possono collocare la biblioteca civica, il laboratorio, il museo, il teatro, l'atelier, l'incubatore di impresa e analoghe iniziative extracurricolari aperte alla cittadinanza. A livello macro si situano i progetti di sensibilizzazione e di intervento ambientale, culturale e sociale $\mathrm{e}$, più in generale, tutte quelle iniziative rivolte al territorio che hanno effetti sulle politiche locali e che influiscono su atteggiamenti e valori della popolazione di riferimento. Le iniziative che si collocano in questo quadrante fanno della scuola un centro di aggregazione sociale e culturale, in cui anche i genitori e i cittadini in genere sono coinvolti contribuendo come esperti - singolarmente o organizzati in associazioni - alle attività formative della scuola, oppure partecipando come fruitori e destinatari di iniziative di formazione proposte in orari che non coincidono necessariamente con quelli scolastici. Trattasi di una scuola aperta, che oltre ad estendere gli orari, mette alcuni dei propri spazi a disposizione del quartiere allestendo strutture permanenti

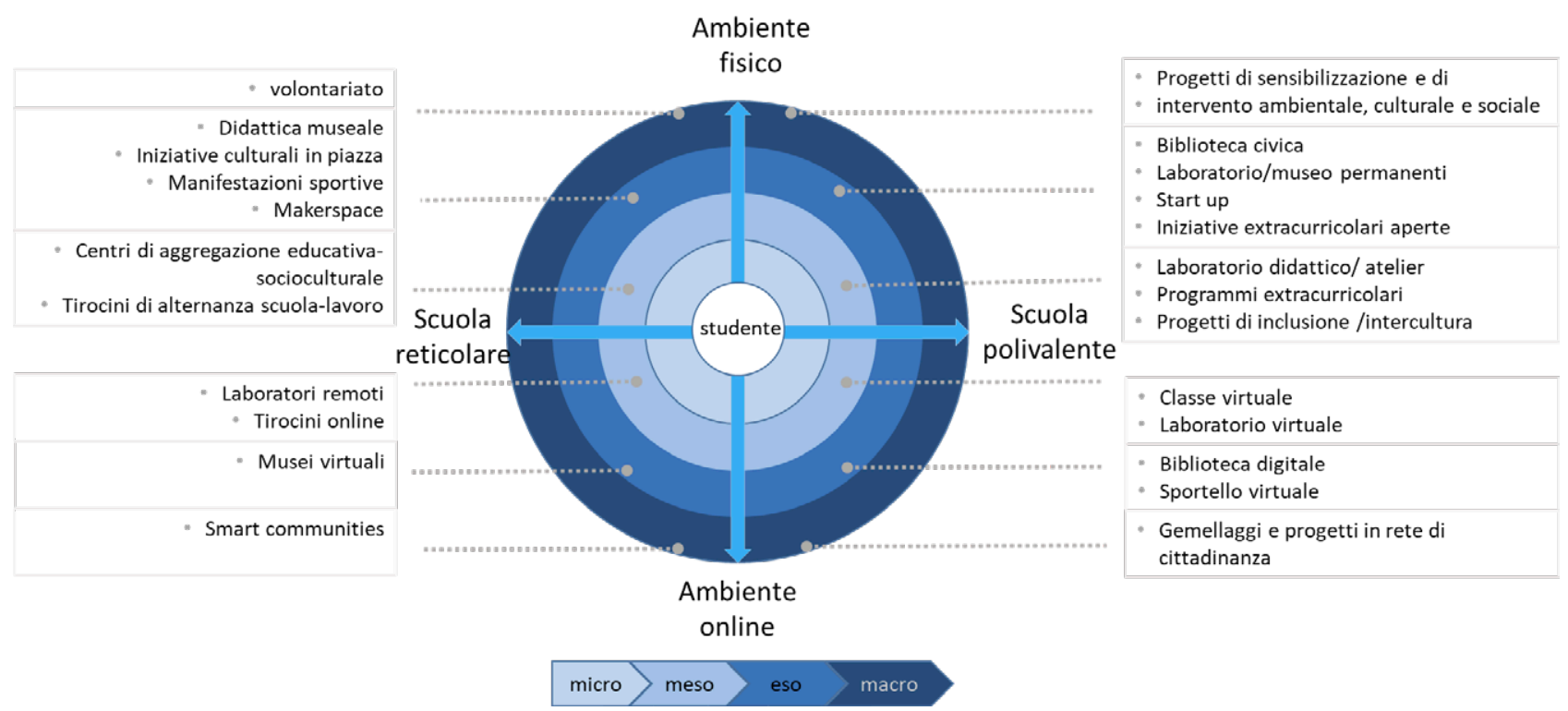

Figura 3. Una mappatura delle possibili istanze di iniziative in collaborazione fra scuola e comunità locale. 
e flessibili che, di norma, non si trovano in un edificio scolastico. Talvolta tali strutture trovano collocazione in spazi inutilizzati della scuola che vengono recuperati nonché, grazie alle attività in essi promosse rispondenti alle esigenze delle famiglie e della cittadinanza, trasformati in un bene culturale comune e in un modello virtuoso di cittadinanza attiva. Tutte le iniziative che rientrano in questo quadrante trovano difficoltà ad essere portate avanti nei periodi di emergenza in cui la scuola, pur essendo aperta, dà accessi contingentati e in orari diversificati agli studenti e al personale scolastico. In questo frangente negli edifici scolastici non possono accedere le famiglie e tanto meno soggetti esterni quali associazioni, volontari, esperti o comuni cittadini. I progetti che coinvolgono utenti esterni o che si rivolgono a destinatari diversi dagli studenti, non possono dunque essere realizzati finché non cessa lo stato di emergenza.

Il secondo quadrante, circoscritto dai semiassi scuola polivalente/ ambiente online, comprende le iniziative promosse dalla scuola, che può avvalersi della collaborazione con entità territoriali, gestite prevalentemente in ambienti online, accessibili dal web. Tali ambienti abbattono i confini spaziali e temporali dell'edificio scolastico mentre, sul piano didattico, possono amplificare i processi di apprendimento sul piano cognitivo e su quello sociale. Le strutture fisiche precedentemente elencate per il primo quadrante, trovano un potenziale corrispettivo nella loro trasposizione virtuale: ricadono quindi in quest'area ambienti quali la classe virtuale per lo svolgimento di attività curricolari ed extracurricolari, la biblioteca digitale, il laboratorio virtuale e lo sportello virtuale, con un impatto più o meno esteso a seconda del livello di coinvolgimento della cittadinanza. L'uso del web moltiplica inoltre i possibili soggetti interlocutori, che non appartengono soltanto alla comunità locale, ma che possono essere dislocati anche in altre città o all'estero: è il caso dell'ambiente virtuale in cui si ritrovano due classi gemellate di paesi diversi che collaborano ad un progetto di cittadinanza attiva, al quale possono contribuire anche associazioni delle rispettive comunità di appartenenza. Nel quadro dell'emergenza Covid-19, tutte le attività inscrivibili in questo quadrante possono essere portate avanti sia nella situazione più estrema, quella di scuola chiusa, sia in condizioni meno restrittive o temporanee, nelle quali sia l'assenza di intere classi, di gruppi di studenti o di singoli può essere gestita attraverso attività di didattica online o tramite forme ibride, che integrano attività online con le lezioni in presenza e che durante l'emergenza attuale sono state sperimentate con esiti positivi nel nostro paese e in molte altre realtà (Cochrane et al., 2020; De Marco, 2020; Dodici et al., 2020; Trentin, 2016).
Il terzo quadrante è demarcato dai semiassi scuola reticolare/ ambiente virtuale ed è quello in cui le possibilità di collaborazione fra scuola e comunità si moltiplicano a dismisura, in quanto le risorse a cui la scuola può attingere non si limitano a quelle offerte dalla comunità locale. Tali risorse open e online possono infatti, grazie alle tecnologie, essere rese disponibili anche da entità che si trovano ovunque, con le quali la scuola stringe partenariati internazionali e costituisce reti collaborative sostenibili. Iniziative esemplificative che ricadono in questo quadrante sono le più svariate: i laboratori remoti (Barry \& Kanematsu, 2020; Evagorou \& Nisiforou, 2020; Kelley, 2020), grazie ai quali gli studenti possono svolgere le simulazioni anche negli ambienti messi a disposizione dagli enti scientifici più prestigiosi; i musei virtuali (Kligler-Vilenchik \& Literat, 2020), visitabili a distanza e comprensivi di attività che si possono svolgere online $o$ in classe; gli ambienti online allestiti dalle imprese per lo svolgimento di tirocini virtuali (Semingson, 2020); le smart communities (Buchholz et al., 2020; Burgess \& Anderson, 2020), che prevedono la collaborazione fra studenti e cittadini per portare a termine progetti dall'elevato valore civico e sociale. Le iniziative che si collocano in questo quadrante amplificano notevolmente le opportunità di apprendimento degli studenti per la forte innovatività, la marcata propensione allo sviluppo sostenibile e per l'eterogeneità dei soggetti che sono potenzialmente coinvolgibili nello sviluppo e nella realizzazione dei percorsi formativi. Tali iniziative sono dunque proponibili anche nelle fasi di emergenza pandemica più spinta, anche perché si svolgono interamente online e non richiedendo la presenza fisica di studenti ed insegnanti a scuola.

Nel quarto quadrante, compreso fra i semiassi scuola reticolare/ ambiente fisico, rientrano tutte le iniziative che la scuola promuove sul territorio, in collaborazione con i vari soggetti della comunità locale. In mancanza di propri spazi da utilizzare, la scuola sfrutta quelli presenti sul territorio e delocalizza in quei luoghi le attività disciplinari o extracurricolari che richiedono spazi e strutture adeguate, dando la propria adesione a progetti promossi dalle varie entità territoriali o collaborando con esse nell'attivazione di nuove iniziative congiunte. Rientrano in questo quadrante attività come quelle di didattica museale, le esperienze di maker education condotte nei FabLab e nei makerspace (Dougherty, 2016; Vuorikari, et al., 2019), le iniziative culturali in piazza, le manifestazioni sportive, i tirocini di alternanza scuola-lavoro, le attività in centri di aggregazione giovanile e socio-educativi e le azioni di volontariato. La situazione pandemica pone dei limiti alle attività classificabili in questo quadrante, che tendono a coincidere con i vin- 
coli che le misure d'emergenza prevedono per gli istituti scolastici: anche nelle strutture presenti sul territorio le misure prevedono numeri contingentati di persone, registrazione degli accessi, regole per limitare la diffusione del virus che spesso impediscono lo svolgimento di un'attività come quelle che richiedono la manipolazione di oggetti, l'utilizzo di uno stesso strumento o di una postazione da parte di diversi studenti. Anche per gli spazi all'aperto come le piazze o i cortili vigono analoghe norme legate al divieto di assembramento. Tuttavia, iniziative di rilievo svolte in questo periodo con numeri contenuti di studenti, come alcune esperienze di outdoor education (Bonell et al., 2020; Quay et al., 2020; Tal et al., 2019) lasciano aperti alcuni spiragli sull'offerta educativa del territorio, che può fornire un supporto alla scuola e sollevarla dalle problematiche relative alla gestione degli spazi.

\section{CONCLUSIONI}

Le mutate condizioni dovute all'era pandemica del COVID-19, così come le recenti sollecitazioni derivanti dall'attuazione delle strategie di sostenibilità dettate dall'Agenda 2030, impongono una revisione dei contenuti curricolari e un riadeguamento dei saperi, che la scuola dovrebbe veicolare attraverso compiti autentici e contestualizzati, percepiti come significativi dagli studenti del terzo millennio. Il compito della scuola non si limita all'insegnamento e la scuola non può essere la sola ad avere questo compito: la comunità non può più fungere da spettatrice o da pubblico ma, ora più che mai, attraverso le associazioni, le imprese, le università e i soggetti che ne fanno parte, deve sentirsi responsabilizzata nel prendersi carico dell'apprendimento dei suoi giovani appartenenti e nel contribuire attivamente ai loro percorsi formativi. Anche le famiglie, che durante il lockdown hanno svolto nel nostro paese un ruolo essenziale, dal punto di vista educativo e del supporto alla didattica formale, possono essere considerate risorse insostituibili con le quali la scuola deve dialogare e collaborare.

È necessario condividere un approccio che riunisca tutte le risorse che si trovano nelle scuole e nelle comunità perché le prime possano attivare delle collaborazioni proficue con le seconde. In questo arduo compito, i soggetti pubblici e privati possono intervenire in aiuto della scuola e sopperire ad essa, integrandone l'offerta educativa. Assumendo una visione sistemica, la scuola può, con l'aiuto degli attori presenti sul territorio, attivarsi per fungere da fulcro delle varie iniziative socioeducative: il modello ecologico definito in questo contributo pone lo studente al centro del sistema e considera le interconnessioni fra le varie strutture sociali implicate nella sua formazione, prospettando diversi scenari che, indipendentemente dall'approccio adottato, conferiscono alla scuola un ruolo propulsivo. In questa cornice lo studente può cogliere l'opportunità di diventare un cittadino attivo e responsabile che vive esperienze di apprendimento significativo, percependo al contempo il proprio essere utile e parte di una comunità coesa e vitale. Le tecnologie e i media educativi, se intesi come totalmente connessi con l'approccio metodologico impiegato al punto da divenire connaturati allo stesso ambiente di apprendimento che contribuiscono a definire; se percepiti come trasparenti da parte di insegnanti e studenti e non come una sovrastruttura aggiunta ed opzionale, possono diventare un elemento potenziante, in senso non solo strumentale quanto strutturale, apportando un contributo essenziale alla trasformazione dei processi di insegnamento e di apprendimento sollecitata dalla situazione critica che stiamo vivendo. In questo senso, attivando attività di networking con il territorio e attuando una trasformazione dei processi educativi indotta dall'uso di approcci didattici innovativi e potenziata da tecnologie e media educativi, la scuola può promuovere azioni didattiche ed educative di qualità, in linea con gli obiettivi di sviluppo sostenibile che allo stato attuale è necessario porsi come traguardi da raggiungere al più presto.

\section{BIBLIOGRAFIA}

AAVV (2012). Indicazioni nazionali per il curricolo della scuola dell'infanzia e del primo ciclo d'istruzione, Annali della Pubblica Istruzione, numero speciale. Le Monnier.

Abbott, J. (2014). Battling for the Soul of Education. The 21st Century Learning Initiative. https://files.eric. ed.gov/fulltext/EJ1077082.pdf

Acton, R. (2017). Place-people-practice-process: Using sociomateriality in university physical spaces research. Educational Philosophy and Theory, 49(14), 1441-1451. https://doi.org/10.1080/00131857.2017.13 09637

Barry, D. M., \& Kanematsu, H. (2020). Teaching during the COVID-19 Pandemic. Online Submission. https:// files.eric.ed.gov/fulltext/ED606017.pdf

Bevan, B., Bell, P., Stevens, R. \& Razfar, A. (Eds.) (2013). LOST opportunities: Learning in out-of-school time. Springer. https://doi.org/10.1007/978-94-007-4304-5

Bonell, C., Melendez-Torres, G. J., Viner, R. M., Rogers, M. B., Whitworth, M., Rutter, H., \& Patton, G. (2020). An evidence-based theory of change for reducing SARS-CoV-2 transmission in reo- 
pened schools. Health \& place, 102398. https://doi. org/10.1016/j.healthplace.2020.102398

Bronfenbrenner, U. (1979). The Ecology of Human Development. Harvard University Press.

Bronfenbrenner, U. (1987). Family support: The quiet revolution. In S. L. Kaagan, D. R. Powell, B. Weissbourd \& E.F. Ziegler (eds.), America's family support programs. Perspectives and prospects (pp. xi-xvii). Yale University Press.

Bronfenbrenner, U. (1990). Discovering what families do. In D. Blankenhorn, S. Bayme \& J.B. Elshtain (eds.), Rebuilding the nest (pp. 27-38). Family Service America.

Buchholz, B. A., DeHart, J., \& Moorman, G. (2020). Digital Citizenship During a Global Pandemic: Moving Beyond Digital Literacy. Journal of Adolescent \& Adult Literacy, 64(1), 11-17. https://doi.org/10.1002/ jaal.1076

Burgess, W. K., \& Anderson, J. L. (2020). Leveraging Community Partnerships to Engage Digitally Foreign Learners in Response to COVID-19. Middle Grades Review, 6(2), 10. https://scholarworks.uvm.edu/mgreview/vol6/iss $2 / 10$

Cahapay, M. B. (2020). Rethinking Education in the New Normal Post-COVID-19 Era: A Curriculum Studies Perspective. Aquademia, 4(2), ep20018. https://doi. org/10.29333/aquademia/8315

Chrzanowski, D., Rans, S. \& Thompson, R. (2010). Building Mutually-Beneficial Relationships Between Schools and Communities: The Role of a Connector. ABCD Institute.

Cochrane, T., Birt, J. R., Cowie, N., Deneen, C., Goldacre, P., Narayan, V., Ransom, L., Sinfield, D., \& Worthington, T. (2020). Designing hybrid practice-based learning environments to facilitate distributed learning communities (COVID-19). In ASCILITE 2020: 37 th International Conference on Innovation, Practice and Research in the Use of Educational Technologies in Tertiary Education (pp. 1-5). Australasian Society for Computers in Learning in Tertiary Education.

Daniel, S. J. (2020). Education and the COVID-19 pandemic. Prospects, 1-6. https://doi.org/10.1007/s11125020-09464-3

De Marco, L. (2020). Dalla DaD alla DDI: esperienze e riflessioni per un modello didattico ibrido-flessibile. Bricks, 4, 132-143. http://www.rivistabricks.it/wpcontent/uploads/2020/09/BRICKS_4_2020.pdf

Dodici, S., Reyes, M. C., \& Trentin, G. (2020). I-MOOC, un mooc interattivo personalizzabile nei tempi e nelle sequenze di fruizione dei contenuti: lopinione dei partecipanti. Italian Journal of Educational Technology, 28(2), 121-137. https://doi. org/10.17471/2499-4324/1154
Dougherty, D. (2016). Free to make: How the maker movement is changing our schools, our jobs, and our minds. North Atlantic Books.

Engeström, Y. (1991). Non scolae sed vitae discimus: Toward overcoming the encapsulation of school learning. Learning and Instruction, 1, 243-259. https://doi.org/10.1016/0959-4752(91)90006-T

Evagorou, M., \& Nisiforou, E. (2020). Engaging Preservice Teachers in an Online STEM Fair during COVID-19. Journal of Technology and Teacher Education, 28(2), 179-186. https://www.learntechlib.org/ primary/p/216234/

Galdieri, M., Todino M. D., Scarinci, A. (2020). (2020). Flessibilità e adattamento al cambiamento nella trasposizione didattica a distanza. Education Sciences \& Society-Open Access, 11(1), 477-503. https://doi. org/10.3280/ess1-2020oa9936

Garcia, A. (ed.), 2014. Teaching in the Connected Learning Classroom. Digital Media and Learning Research Hub. https://dmlhub.net/wp-content/uploads/files/ teaching-in-the-CL-classroom.pdf

Gee, J. P. (2003). What video games have to teach us about learning and literacy. Computers in Entertainment (CIE), 1(1), 20-20. https://doi.org/10.1145/950566.950595

Hull, G., \& Schultz, K. (2001). Literacy and learning out of school: A review of theory and research. Review of Educational Research, 71(4), 575-611. https://doi. org/10.3102\%2F00346543071004575

Imms, W., Mahat, M., Byers, T., \& Murphy, D. (2017). Type and use of innovative learning environments in Australasian schools. ILETC Survey 1. https://files. eric.ed.gov/fulltext/ED577584.pdf

Ito, M., Baumer, S., Bittanti, M., Boyd, D., Cody, R., HerrStephenson B., Horst, H. A., Lange, P. G., Mahendran, D., Martinez, K. Z., Pascoe, C. J., Perkel, D., Robinson, L. , Sims, C., \& Tripp, L. (2009). Hanging Out, Messing Around, and Geeking Out: Kids Living and Learning with New Media. The MIT Press. https://doi.org/10.7551/mitpress/8402.001.0001

Ito, M., Gutiérrez, K., Livingstone, S., Penuel, B., Rhodes, J., Salen, K., Schor, J., Sefton-Green, J. \& Watkins, S. C. (2013). Connected learning: an agenda for research and design. Digital Media and Learning Research Hub. Digital Media and Learning Research Hub.

Iyengar, R. (2020). Education as the path to a sustainable recovery from COVID-19. Prospects, 49, 77-80. https://doi.org/10.1007/s11125-020-09488-9

Kelley, E. W. (2020). Reflections on Three Different High School Chemistry Lab Formats during COVID-19 Remote Learning. Journal of Chemical Education, 97(9), 2606-2616. https://doi.org/10.1021/acs. jchemed.0c00814 
Kligler-Vilenchik, N., \& Literat, I. (2020). Youth Digital Participation: Now More than Ever. Media and Communication, 8(2), 171-174. https://doi.org/10.17645/ mac.v8i2.3180

Livari, N., Sharma, S., \& Ventä-Olkkonen, L. (2020). Digital transformation of everyday life-How COVID-19 pandemic transformed the basic education of the young generation and why information management research should care?. International Journal of Information Management, 102183. https://doi. org/10.1016/j.ijinfomgt.2020.102183

Marcarini, M. (2014). Gli spazi della scuola: le proposte rivoluzionarie dell'attivismo nell'organizzazione degli spazi educativi e le ricadute successive. Formazione, lavoro, persona. 4(10), 3. https://forperlav.unibg.it/ index.php/fpl/article/view/137/117

Mulcahy, D., Cleveland, B., \& Aberton, H. (2015). Learning spaces and pedagogic change: Envisioned, enacted and experienced. Pedagogy, Culture \& Society, 23(4), 575-595. https://doi.org/10.1080/14681366.201 5.1055128

Nussbaum Beach, S. \& Ritter Hall, L., (2011). The Connected Educator. Solution Tree Press. https://doi.org/1 0.1080/08923647.2013.757065

Paone, F. (2020). Learning Environment as Bridge Between School and Community. In J. L. Sarasola Sánchez-Serrano, F. Maturo \& Š. Hošková-Mayerová (eds.), Qualitative and Quantitative Models in SocioEconomic Systems and Social Work (pp. 151-159). Springer, Cham. https://doi.org/10.1007/978-3-03018593-0_12

Quay, J., Gray, T., Thomas, G., Allen-Craig, S., Asfeldt, M., Andkjaer, S., \& Ho, S. (2020). What future/s for outdoor and environmental education in a world that has contended with COVID-19?. Journal of Outdoor and Environmental Education, 23(2), 93-117. https:// doi.org/10.1007/s42322-020-00059-2

Repetto, M. (2013). Networked informal learning and continuing teacher education. In G. Trentin \& M. Repetto (eds.), Using Network and Mobile Technology to Bridge Formal and Informal Learning, 183-207, Elsevier. https://doi.org/10.1016/B978-1-84334-6999.50007-0

Resnick, L. B. (1987). The 1987 Presidential Address: Learning in school and out. $E d u$ cational Researcher, 16(9), 13-20. https://doi. org/10.3102\%2F0013189X016009013

Roth, S. \& Erstad, O. (2013). Networked lives for learning: digital media and Young practices across formal and informal contexts. In G. Trentin \& M. Repetto (eds.), Using Network and Mobile Technology to Bridge Formal and Informal Learning, 119-152, Else- vier. https://doi.org/10.1016/B978-1-84334-6999.50005-7

Roth, W.-M., \& Lee, Y. J. (2006). Contradictions in theorizing and implementing "communities". Educational Research Review, 1(1), 27-40. Amsterdam: Elsevier. https://doi.org/10.1016/j.edurev.2006.01.002

Sahlberg, P. (2011). Finnish Lessons 2.0. Columbia University: Teachers College.

Save the Children Italia (2012). Fare comunità educante: la sfida da vincere. Crescere al Sud. https://scienzepolitiche.unical.it/bacheca/archivio/materiale/99/ minori/IMP-crescere-al-sud.pdf (ver. 12.10.2020).

Selwyn, N. (2011). Finding an Appropriate fit for me: Examining the (in)Flexibilities Of International Distance Learning. International Journal of Lifelong Education, 30(3), 367-383. https://doi.org/10.1080/026013 70.2011 .570873

Semingson, P. (2020). Cross-Course Mentoring via a Shared Communal Blog: Best Practices and Lessons Learned. In Society for Information Technology \& Teacher Education International Conference (pp. 2119-2124). Association for the Advancement of Computing in Education (AACE). https://www.learntechlib.org/primary/p/216082/

Siemens, G. (2004). Connectivism: A Learning Theory for the Digital Age. Elearnspace.org. http://citeseerx.ist.psu.edu/viewdoc/download? $\mathrm{doi}=10.1 \cdot 1.1089 .2000 \& \mathrm{rep}=\mathrm{rep} 1 \&$ type $=\mathrm{pdf} \quad($ ver. 12.10.2020).

Singal, N., \& Swann, M. (2011). Children's perceptions of themselves as learner inside and outside school. Research Papers in Education, 26(4), 469-484. https:// doi.org/10.1080/02671520903281617

Tal, T., Levin-Peled, R., \& Levy, K. S., (2019). Teacher views on inquiry-based learning: the contribution of diverse experiences in the outdoor environment. Innovation and Education, 1(1), 2. https://doi. org/10.1186/s42862-019-0004-y

Tosi, L. (2019). Proposte e azioni per progettare, allestire e utilizzare spazi flessibili a scuola. Scuola democratica, 10(2), 409-416. https://doi.org/10.12828/94823

Trentin, G. (2016). Always-on education and hybrid learning spaces. Educational Technology, 56(2), 31-37. http://www.jstor.org/stable/44430457

Vuorikari, R., Ferrari, A., \& Punie, Y. (2019). Makerspaces for Education and Training - Exploring future implications for Europe. EUR 29819 EN, Publications Office of the European Union. JRC117481. https://doi. org/10.2760/946996 